\title{
Prospective randomised clinical trial of intravitreal bevacizumab versus triamcinolone in eyes with diabetic macular oedema undergoing cataract surgery: 6-month results
}

\author{
Rathika Kandasamy (1) , Marios Constantinou, Sophie L Rogers (ㄷ) , \\ Sukhpal Singh Sandhu, Sanjeewa Wickremasinghe, Salmaan Al-Qureshi, Lyndell L Lim
}

Department of Surgery (Ophthalmology), Centre for Eye Research Australia, Royal Victorian Eye and Ear Hospital, University of Melbourne, East Melbourne, Victoria, Australia

\section{Correspondence to} Dr Lyndell L Lim, Department of Surgery (Ophthalmology), Centre for Eye Research Australia, University of Melbourne, Royal Victorian Eye and Ear Hospital, East Melbourne, Victoria, Australia; limllp@unimelb.edu.au

Received 23 October 2018 Revised 31 January 2019 Accepted 31 January 2019 Published Online First 28 February 2019
Check for updates

(C) Author(s) (or their employer(s)) 2019. No commercial re-use. See rights and permissions. Published by BMJ.

To cite: Kandasamy $R$, Constantinou M, Rogers SL, et al. Br J Ophthalmol 2019;103:1753-1758

\section{ABSTRACT}

Aim To report the 6-month results of a clinical trial that compared intravitreous bevacizumab (BVB)

$1.25 \mathrm{mg}$ versus triamcinolone acetonide ( $T A$ ) $4 \mathrm{mg}$ when administered as an adjunct during cataract surgery to patients with diabetic macular oedema (DMO).

Methods Prospective, double-masked, single-centre (Royal Victorian Eye and Ear Hospital, Melbourne) clinical trial. Patients with visually significant cataract and centreinvolving DMO (either current or prior) were randomised (1: 1) to receive either intravitreous BVB $1.25 \mathrm{mg}$ or TA $4 \mathrm{mg}$ at the time of cataract surgery and if required at review. Main outcome measures were changes in best-corrected visual acuity (BCVA) and central macular thickness (CMT) from baseline to the 6 -month time point of this 12-month study.

Results 61 eyes of 58 patients were enrolled. At baseline, both groups were similar in terms of BCVA and CMT ( $p>0.2)$. At 6 months, there was no significant difference in vision between the groups, with mean letter gain of $+21.4(95 \% \mathrm{Cl}+14.5$ to +28.4$)$ in the TA group and $+17.3(95 \% \mathrm{Cl}+12.1$ to +22.6$)$ in the BVB group ( $p=0.35$ ). The TA group had a significant sustained anatomical improvement at 6 months, with a reduction in CMT $(-51.4 \mu \mathrm{m} ; 95 \% \mathrm{Cl}-98.2$ to -4.7$)$ compared with thickening in the BVB group $(+15.6 \mu \mathrm{m} ; 95 \% \mathrm{Cl}$ -26.4 to $+57.7, p=0.04)$.

Conclusions When given as an adjunct to cataract surgery, both TA and BVB improved visual outcomes at 6 months postoperatively. However, only TA resulted in sustained improvement in CMT, with the majority not requiring any further treatment postoperatively.

\section{INTRODUCTION}

Diabetic macular oedema (DMO) is a leading cause of visual impairment in patients with diabetic retinopathy. ${ }^{1}$ Multiple studies have suggested a correlation between poor postoperative visual outcomes and the presence of macular oedema at the time of cataract surgery. ${ }^{2-5}$ Alterations to the ocular microenvironment that occur after cataract surgery are also thought to promote the development of DMO. ${ }^{6}$ Cataract surgery in diabetics has been demonstrated to cause an imbalance between angiogenic and antiangiogenic growth factors and result in elevated levels of inflammatory cytokines, thereby potentially leading to changes that induce or cause progression of DMO. ${ }^{6}$ As the pathogenesis of DMO is not purely vascular endothelial growth factor (VEGF) driven, steroids rather than antiVEGF agents may be a better option for the treatment of DMO, ${ }^{7}$ as they have both anti-inflammatory and anti-VEGF effects. ${ }^{8}$ Although there have been studies that compared the effect of anti-VEGF agents versus triamcinolone on DMO, ${ }^{9-12}$ this study is the first prospective, randomised trial that directly compares these two modalities for the treatment of DMO in the setting of cataract surgery. ${ }^{13}$

The Diabetic Macular Edema at the time of Cataract Surgery Trial (DIMECAT) is designed to compare the differences at 12 months in visual and anatomical outcomes between intravitreous bevacizumab (BVB, Genentech, San Francisco, California, USA; $1.25 \mathrm{mg}$ ) and triamcinolone acetonide (TA, Alcon Pharmaceuticals, Fort Worth, Texas, USA; 4 $\mathrm{mg}$ ) when administered as an adjunct at the time of cataract surgery, then as needed on review, to patients with DMO undergoing cataract surgery. This paper reports the 6-month results of the primary outcome measures of this trial: changes in best-corrected logarithm of the minimum angle of resolution (logMAR) visual acuity (BCVA) and central macular thickness (CMT) from baseline to the 6-month time point.

\section{METHODS}

The methodology of this prospective, randomised (allocation ratio 1:1), double-masked, paralleltreatment trial has been described in a previous report. ${ }^{13}$ Eligible patients with visually significant cataract and centre-involving DMO, either current or within the last 24 months, were recruited from the Medical Retina Clinic of the Royal Victorian Eye and Ear Hospital (Melbourne, Australia) (RVEEH). Patients with macular ischaemia or other potential causes of macular oedema were excluded (refer online supplementary table 1). ${ }^{13}$ Participants were block randomised to receive either intravitreous BVB or TA at the time of cataract surgery after providing written informed consent. The trial adhered to the tenets of the Declaration of Helsinki.

Biometry and standard ophthalmic examinations were performed on all patients prior to surgery. All patients underwent standard phacoemulsification and AcrySof SA60AT (Alcon, Fort Worth, Texas, USA) intraocular lens (IOL) implantation followed 
by intravitreous injection of either randomly assigned agent. ${ }^{13}$ All patients were treated with a standard regimen of postoperative drops four times a day (1\% prednisolone acetate (Prednefrin Forte, Allergan, Dublin, Ireland) and chloramphenicol $0.5 \%$ (Chlorsig, Sigma Pharmaceuticals, Victoria, Australia)) tapered over 4 weeks. In the event of complicated surgery, the patient was withdrawn from the trial with no study treatment being administered.

Participants were examined at baseline, 1 week postsurgery and monthly thereafter for 12 months. Clinical characteristics and demographic details were recorded at baseline. ${ }^{13}$ At each visit, patients underwent a complete ophthalmic examination including assessment of BCVA, intraocular pressure (IOP) and CMT measurement (Spectralis ocular coherence tomography (OCT)) (Heidelberg Engineering, Heidelberg, Germany). Primary outcome measures were assessed by masked examiners and participants were masked to their study treatments throughout the duration of the trial.

Topical antiglaucoma medications were commenced if IOP measurements were greater than $30 \mathrm{~mm} \mathrm{Hg}$ on a single reading or $21 \mathrm{~mm} \mathrm{Hg}$ on two consecutive visits. ${ }^{13}$ Retreatment was performed if there was a loss of five or more letters of BCVA or a $50 \mu \mathrm{m}$ increase in CMT compared with previous bestrecorded results. ${ }^{13}$ Treatment failure was defined as failure to show response or clinical worsening despite receiving study treatments. ${ }^{13}$ Alternative treatment strategies were commenced in these cases.

\section{Statistical analysis}

Data from the first 6 months from all eligible participants has been included in this intention-to-treat analysis. The target sample size for this study was calculated as 46 eyes per treatment (calculations reported previously ${ }^{13}$ ). Unforeseen increases in the waiting time for surgery at the RVEEH occurred after the first 9 months of recruitment leading to many patients withdrawing prior to randomisation to have their surgery performed earlier, elsewhere. Enrolment into the trial was eventually ceased earlier than anticipated after 30 months because of the high rate of subject withdrawals, and the resultant unsustainable extension to the overall trial duration. Of those that remained in the study through to 6 months, complete follow-up was achieved in 39 $(64 \%)$ eyes and two consecutive visits were missed by only two eyes (3\%). Missingness was investigated and found to be independent of outcome measures at the previous visit. Additionally, baseline characteristics were not different in those subjects with any missing outcome values compared with those with complete follow-up. As such, missingness was determined to be ignorable and observed values only were utilised in the mixed model analysis. ${ }^{14}$

Continuous variables were summarised as means with 95\% CI or as medians with IQR if they did not appear to be normally distributed. Baseline characteristics were compared across treatment groups using two-sample t-test (with or without unequal variance as needed) or Mann-Whitney U test, respectively. Categorical variables were summarised as percentages and compared using Fisher's exact test.

The main outcomes of interest (BCVA and CMT) were examined separately using a multilevel mixed-effects linear regression model with random intercept and random slope for time and unstructured covariance. Mixed models were constructed utilising all available observed data points. ${ }^{14}$ Deviance statistics and Akaike Information Criterion were compared to determine whether a more complex model was a better fit to the data.

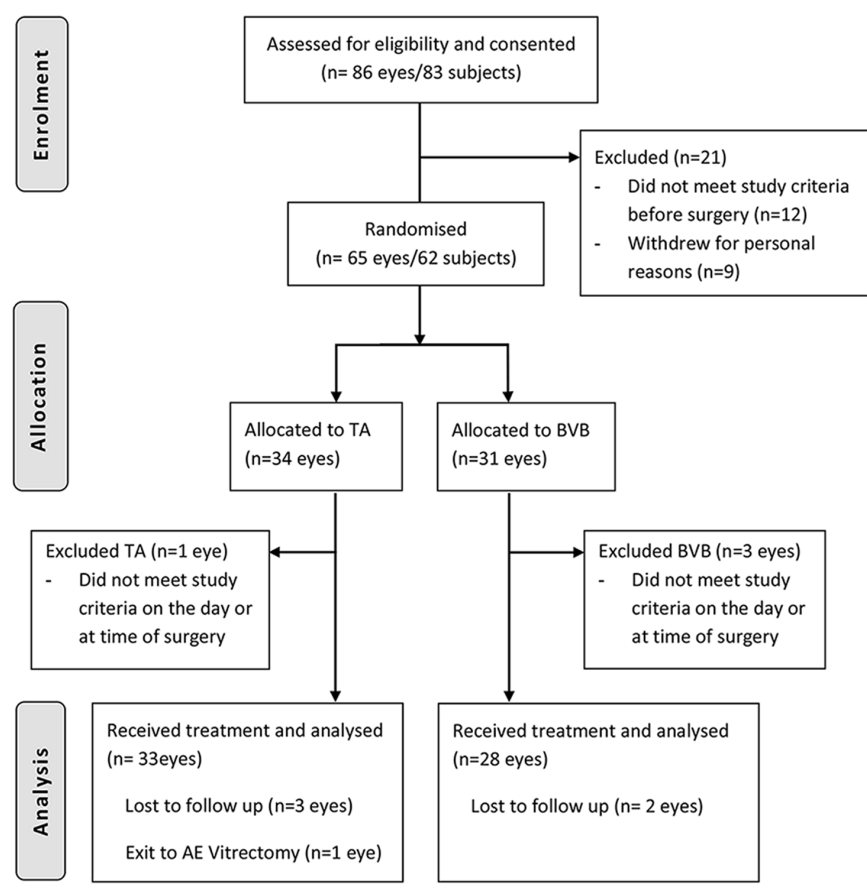

Figure 1 Study design flow diagram baseline to month 12. BVB, bevacizumab; TA, triamcinolone.

The final models include baseline BCVA (or CMT), interaction terms for treatment and time and for DMO status at enrolment and time and three levels to account for the nesting of repeated measurements over time within eyes and eyes within subjects. CMT mixed models were run including and excluding all data from the one eye with an extreme outlying value at baseline.

All analyses were undertaken using Stata IC V.14.2 for Windows.

\section{RESULTS}

Between June 2012 and August 2017, 86 eyes of 83 patients were enrolled. Of these, 21 patients were excluded prior to randomisation as they did not meet the study criteria at baseline $(n=12)$ or withdrew for personal reasons $(n=9)$, with the longer than expected waiting period for surgery quoted as a major factor. Four other patients were exited at the time of surgery due to intraoperative complications unrelated to the therapeutic interventions necessitating withdrawal $(n=3)$ or electing to have surgery privately $(n=1)$. The remaining 58 patients (61 eyes; 33 TA group, 28 BVB group) received treatment according to study protocol and are analysed herein (refer figure 1). Six participants $(10 \%)$ were lost to follow-up before the 6-month time point: four withdrew citing personal reasons (two TA, two BVB), one had a concurrent medical illness that precluded attending monthly follow-ups (TA) and the remaining patient underwent a vitrectomy for vitreous haemorrhage secondary to proliferative diabetic retinopathy and was exited at month 4 (TA). Three patients had both eyes enrolled (two had both eyes assigned to TA and one had an eye enrolled in each treatment arm).

Baseline characteristics were similar between treatment groups, except for age, where patients allocated to BVB were older than those in the TA group (70.2 years and 64.3 years, respectively, $\mathrm{p}=0.007$ ) (table 1). There was no significant difference in BCVA or CMT between the groups at baseline $(\mathrm{p}>0.2)$. The majority of eyes had current DMO (79\%), and more than half (56\%) had received previous treatment for $\mathrm{DMO}$, whether macular laser (13 BVB, $18 \mathrm{TA}$ ) and/or intravitreal injections (four BVB, three 


\begin{tabular}{|c|c|c|}
\hline Characteristics per person & BVB ( $n=28$ eyes) & TA ( $n=33$ eyes) \\
\hline $\begin{array}{l}\text { Age* }^{*} \text { at the time of surgery, } \\
\text { mean }(95 \% \mathrm{Cl}) \text {, years }\end{array}$ & $70.2(67.4-73.0)$ & $64.3(61.1-67.5) \dagger$ \\
\hline Males $\ddagger$ n (\%) & $18(64)$ & $24(73)$ \\
\hline $\begin{array}{l}\text { Baseline HbA1c§, median } \\
\text { (IQR),\% }\end{array}$ & $7.5(7-8.6)$ & $7.5(6.3-8.4)$ \\
\hline \multicolumn{3}{|l|}{ Diabetes $\neq, \mathrm{n}(\%)$} \\
\hline Type 1 & 0 & $1(3)$ \\
\hline Type 2 , requiring insulin & $17(61)$ & $21(64)$ \\
\hline Type 2, not requiring insulin & $11(39)$ & $11(33)$ \\
\hline \multicolumn{3}{|l|}{ Characteristics per study eye } \\
\hline Left eyes, No. (\%) & $10(36)$ & $15(46)$ \\
\hline $\begin{array}{l}\text { BCVA baseline, mean }(95 \% \mathrm{Cl}) \text {, } \\
\text { letters }\end{array}$ & $55.1(48.7-61.4)$ & $50.5(45.3-55.8)$ \\
\hline $\begin{array}{l}\text { CMT baseline, median (IQR), } \\
\text { microns }\end{array}$ & $307.5(277.5-391.5)$ & $316(282-457)$ \\
\hline \multicolumn{3}{|l|}{ DR severity, No. (\%) } \\
\hline Mild & $1(4)$ & $6(19)$ \\
\hline Moderate & $13(46)$ & $11(33)$ \\
\hline Severe & $3(11)$ & $5(15)$ \\
\hline PRP only (inactive PDR) & $9(32)$ & $11(33)$ \\
\hline Treated PDR (active) & $2(7)$ & 0 \\
\hline DMO at baseline, $n(\%)$ & $22(79)$ & $26(79)$ \\
\hline \multicolumn{3}{|c|}{ Study eye was previously treated, priorf to study entry, $\mathrm{n}(\%)$} \\
\hline $\begin{array}{l}\text { Any treatment (TA, BVB or } \\
\text { macular laser)ף }\end{array}$ & $14(50)$ & $20(61)$ \\
\hline Macular laser & $13(46)$ & $18(55)$ \\
\hline PRP Laser & $10(36)$ & $9(27)$ \\
\hline BVB & $3(11)$ & $3(9)$ \\
\hline TA & $1(4)$ & 0 \\
\hline
\end{tabular}

*The person's age at the time of the eye's surgery.

†Two-sample $t$-test, $p$ value $=0.007$. All other comparisons were not significant ( $>>0.05)$.

$\ddagger$ Count and percentage of eyes in the study that belong to a person with this characteristic.

$\S$ The person's HbA1c levels at the time of the eye's surgery.

IPrevious treatments defined as BVB or TA received within 24 months prior to study entry and macular laser treatment at any time prior to study entry. BCVA, best corrected visual acuity; BVB, bevacizumab; CMT, central macular thickness; DMO, diabetic macular oedema; DR, diabetic retinopathy; HbA1c, haemoglobin A1 c; PDR, proliferative diabetic retinopathy; PRP, panretinal photocoagulation; $\mathrm{TA}$, triamcinolone.

TA). Three patients in the BVB group and one patient in the TA group had received both macular laser and intravitreal injections of either BVB or TA.

At 6 months, the mean BCVA was 71.6 letters $(95 \%$ CI 66.1 to 77.1 ) in the BVB group compared with 72.5 letters $(95 \%$ CI 67.5 to 77.6 ) in the TA group, with no significant difference between groups (mixed model: over 6 months, $p=0.19$; at 6 months $\mathrm{p}=0.41)$. An initial marked improvement in mean BCVA was observed in both treatment arms, followed by more modest increments over the subsequent months (figure 2). Both groups demonstrated a significant improvement from baseline in BCVA at all examinations $(\mathrm{p}<0.05)$; by 6 months, the TA group had a mean letter gain of $+21.4(95 \% \mathrm{CI}+14.5$ to +28.4$)$ compared with $+17.3(95 \% \mathrm{CI}+12.1$ to +22.6$)$ in the BVB group $(\mathrm{p}=0.35)$ (figure 3$)$.

There was a significant difference in CMT between the treatment arms over the 6 months, after accounting for longitudinal effects, nesting, baseline CMT values and DMO status at enrolment (mixed model: over 6 months, $\mathrm{p}=0.002$; at 6 months,

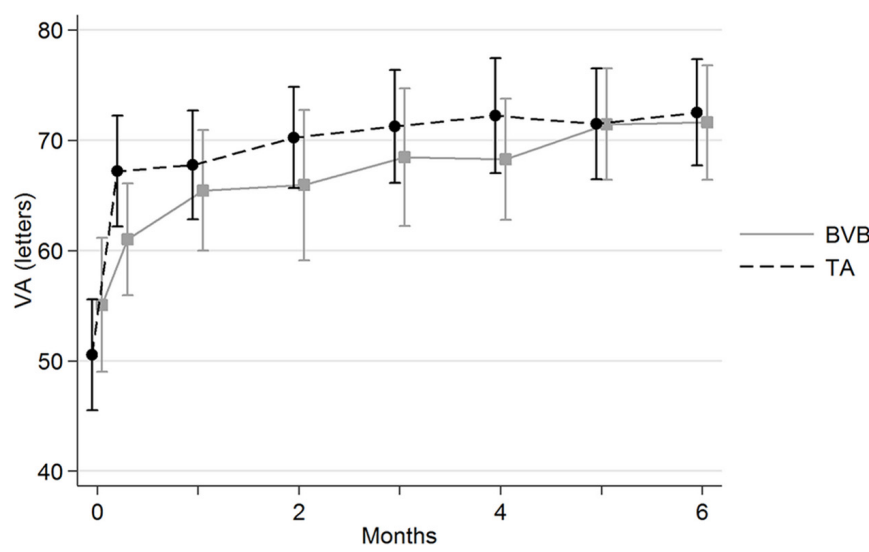

Figure 2 Mean visual acuity at different visits, by treatment. BVB, bevacizumab; TA, triamcinolone; VA, visual acuity.

$\mathrm{p}=0.019)$. This difference remained significant after removing a subject with extreme outlying value of baseline CMT (mixed model: over 6 months, $p=0.002$; at 6 months, $p=0.04$ ). The TA group demonstrated a significant reduction in CMT $(-51.4 \mu \mathrm{m}$ reduction (95\% CI -98.2 to -4.7$)$ ) versus thickening in the BVB group $(+15.6 \mu \mathrm{m}$ increase $(95 \% \mathrm{CI}-26.4$ to +57.7$), \mathrm{p}=0.04)$ (figure 4).

Throughout the first 6 months of the study, the majority of eyes $(25 ; 76 \%)$ in the TA group required no retreatments (mean number of retreatments 0.24 , range $0-1$ ) compared with 12 $(43 \%)$ in the BVB group (mean number of retreatments 1.43 , range $0-6 ; p=0.009$ ) (online supplementary figure 1 ). None of the participants required macular laser throughout the study duration and no patients were classified as treatment failures.

\section{Adverse events}

IOP elevations of more than $21 \mathrm{~mm} \mathrm{Hg}$ were noted in four eyes in the TA group (12\%) and three in the BVB group (11\%; $p>0.99)$ (online supplementary table 2). Of these, three eyes had IOP readings of $25 \mathrm{~mm} \mathrm{Hg}$ or more (all TA group), although none had received retreatments with the study drug. One eye in the TA group required commencement of topical IOP-lowering medications at month 6 (IOP $38 \mathrm{~mm} \mathrm{Hg}$ ). In this eye, the IOP elevation was managed with topical agents (brimonidine tartrate/timolol maleate $0.2 \% / 0.5 \%$ (Allergan, Dublin, Ireland), latanaprost 0.05\% (Pfizer, New York, USA), dorzolamide hydrochloride 2\%

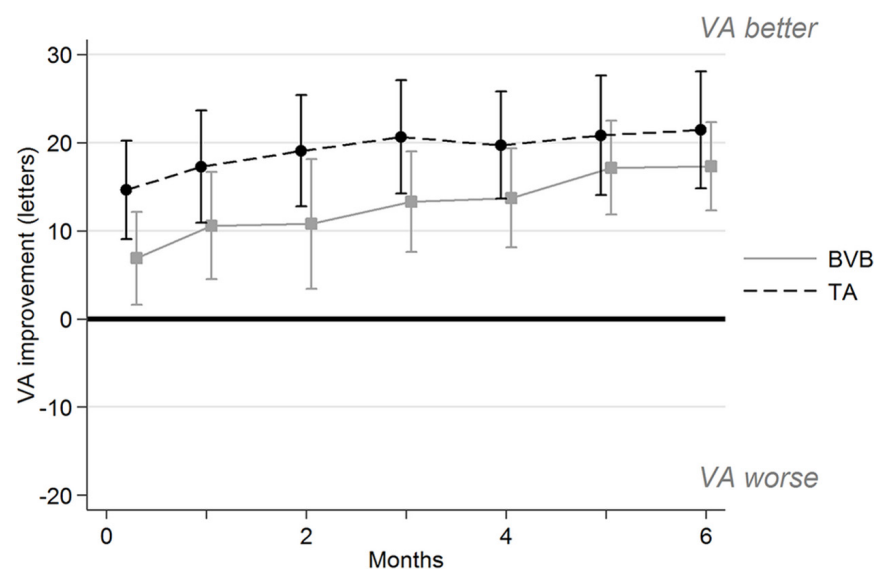

Figure 3 Mean improvement from baseline in visual acuity at different visits, by treatment. BVB, bevacizumab; TA, triamcinolone; VA, visual acuity. 


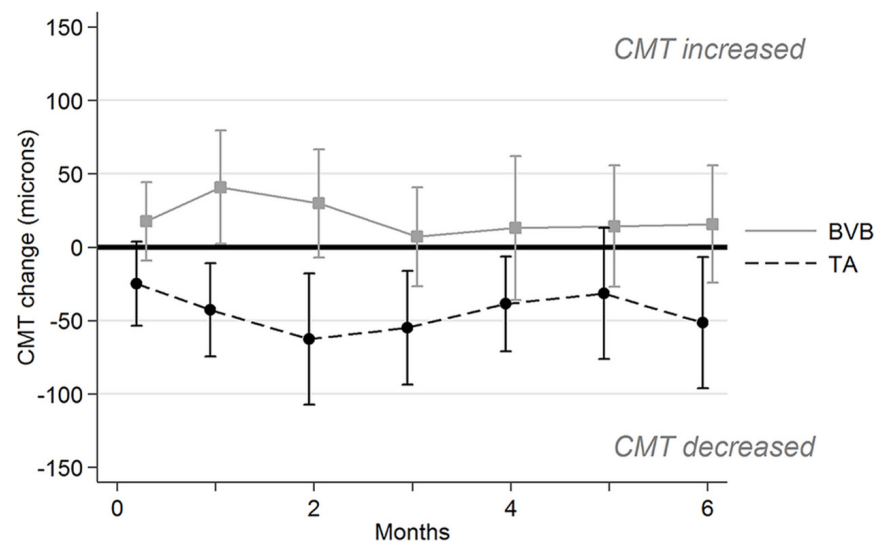

Figure 4 Mean change from baseline in central macular thickness at different visits, by treatment. BVB, bevacizumab; CMT, central macular thickness; TA, triamcinolone.

(Santen Pharmaceutical, Japan)) which were subsequently ceased over the following 4 months with no further rises in IOP after discontinuation of treatment. All other eyes with increased IOP were managed with observation alone. Among eyes that experienced a rise in IOP, only one had received intravitreous treatment prior to baseline, where a single injection of BVB was given 7 months earlier. There were no cases of endophthalmitis or any other serious adverse events attributable to the study treatments in either group.

\section{DISCUSSION}

While there have been previous studies that evaluated the outcome of cataract surgery combined with either $\mathrm{TA}^{15-17}$ or $\mathrm{BVB},{ }^{18-20}$ no studies have directly compared the two modalities in cataract surgery. As such, there is no consensus on which drug would be the optimal agent to use in the management of these patients in the perioperative period. Our study is the first prospective, randomised study to directly compare the use of intravitreous injections of BVB versus TA in the setting of cataract surgery in patients with pre-existing DMO, whether current or past. The 6-month results show that both agents improved visual acuity outcomes, with a trend towards slightly better vision in the TA group. Interestingly, the anatomical benefit was more pronounced in the TA group, which experienced a significant reduction in CMT $(-51.4 \mu \mathrm{m})$ compared with the BVB group, where a slight thickening from baseline $(+15.6 \mu \mathrm{m})$ was seen. This response was noted to be sustained 6 months postoperatively with the majority of TA patients requiring no further retreatments over this time.

The differences in morphological outcomes between the two drugs may be explained by their differing effects on intraocular cytokines. TA has been demonstrated to have a dual effect, inhibiting both inflammatory and angiogenic cytokines, whereas BVB only reduces VEGF, although to a greater extent than TA. ${ }^{21}$ These findings provide further evidence in favour of the pathogenesis of DMO being due to a combination of inflammatory cytokines and not purely VEGF levels, a conclusion that is echoed in results from other groups. ${ }^{22-24}$ Furthermore, since the changes after cataract surgery lead to an ocular microenvironment that is both proinflammatory and proangiogenic, ${ }^{6}$ the dual action of TA in this regard may make it a better option than BVB in this setting.

While TA showed significant morphological improvement compared with BVB, this did not translate into a statistically significant difference in BCVA between the groups, although the results showed slightly better visual outcomes in the TA group. Recent trials on eyes with persistent DMO despite treatment with anti-VEGF have reported similar findings, where switching to or adding corticosteroids significantly reduced macular thickness despite not resulting in further gains in visual acuity. ${ }^{25} 26$ In our study, several factors may account for this finding. First, the sample size may have been insufficient to demonstrate a significant difference in BCVA between the groups. Second, the correlation between macular thickness and visual function in DMO is still unclear, with differing results reported between groups. ${ }^{27-29}$ At best, there is only a modest correlation between central macular thickness and visual acuity, ${ }^{28}$ with a large variability reported between the strength of these associations. ${ }^{30}$ Novel OCT parameters such as outer retinal layer thickness have been demonstrated to correlate better with visual acuity suggesting that specific microstructural changes may be better predictors of visual acuity than central macular thickness. ${ }^{31} 32$ This may account for why the significant anatomical improvement in macular thickness that we found with TA compared with BVB did not translate into better visual outcomes in the TA group.

There was a significant reduction in treatment load in the TA group compared with the BVB group through to the 6-month examination: only $24 \%$ of patients with TA needed retreatment compared with $57 \%$ in the BVB group. While this is not unexpected given the drugs' different half-lives in the eye, it is interesting to note the significantly larger proportion of patients with TA who did not require any further treatments after surgery through to 6 months, which is well beyond the expected duration of action of TA of 77 days. Similar findings have been reported previously with the effect of a single dose of intravitreal TA lasting up to 6 months postoperatively. ${ }^{33}$ Needless to say, the lower number of retreatments in the TA group is a definite advantage given patient convenience/ preferences and the known procedure-related risks such as endophthalmitis.

Intravitreal injections with either agent have similar procedural risks; however, there is an increased risk of IOP elevation $^{15}$ and endophthalmitis ${ }^{34}$ with intravitreal steroids. There were no cases of endophthalmitis in either group and the rate of increased IOP among those in the TA group (12\%) was much lower in our trial than other studies where rates of up to $23.5 \%$ have been described. ${ }^{15} 16$ Contrary to other reports, ${ }^{1018}$ our trial also saw raised IOP in $11 \%$ of eyes in the BVB group, although these IOP elevations were more modest and transient, with no patients having an IOP greater than 25 $\mathrm{mm} \mathrm{Hg}$. While the risk of raised IOP with anti-VEGF injections are less commonly reported, a recent large pharmacoepidemiological study suggested that seven or more injections of BVB annually are associated with increased risk of requiring glaucoma surgery. ${ }^{35}$ This, however, could not explain our findings because among the patients with increased IOP in the BVB group, only one patient had received a single intravitreous injection of $\mathrm{BVB}$ prior to study commencement. As the maximal number of injections in the BVB group at the 6 -month point was only 6 , it remains to be seen if the incidence of raised IOP will increase in this group with further follow-up and potentially more retreatments.

\section{Limitations}

There are some limitations to this study. At baseline, the age between the two treatment groups was statistically different 
and likely the result of randomisation within a small sample group, where it is sometimes difficult to ensure that demographic parameters between treatment arms are similarly distributed. Nonetheless, the actual difference in mean age between groups was only 5.9 years. Sample size was also an important limitation. Despite initially recruiting 86 eyes, a significant proportion of our eligible eyes were excluded prior to randomisation as they did not fulfil study criteria $(n=12)$ or withdrew citing personal reasons $(n=9)$. This was chiefly due to the inherent limitations of a large public hospital setting with significant cataract surgery waiting lists, where the long waiting period allows time for alteration in clinical condition or patients to have a change of heart or seek treatment elsewhere. It is possible that with a larger sample size we may have been able to show a significant difference in BCVA and IOP outcomes between the groups, and our relatively short follow-up of 6 months could be inadequate to demonstrate a significant difference in IOP between the two treatments. It should also be noted that, since slightly more than half the patients in our study had received treatment for DMO (macular laser, intravitreous BVB or TA) prior to cataract surgery, the results reported here may not be applicable to treatment-naïve eyes with DMO at the time of surgery.

\section{CONCLUSIONS}

In conclusion, our 6-month results have demonstrated that cataract surgery augmented with either intravitreous BVB or TA leads to a significant improvement in visual acuity outcomes postoperatively compared with cataract surgery without any other adjunctive measures. ${ }^{15161819}$ These findings confirm the earlier reported outcomes in our preliminary paper ${ }^{13}$ and suggest that the standard of care for all patients with DMO undergoing cataract surgery should be to receive either agent intraoperatively and postoperatively as required. The TA group was found to have a significant reduction in CMT, while the BVB group showed slightly increased CMT despite repeated retreatments. In addition, the majority of the TA group was able to sustain the anatomical improvement in CMT through to the 6 -month time point without requiring retreatments. Although we could not show statistically significant differences in visual function between the groups, a better understanding of the reason behind these differences may be elucidated by detailed analysis of OCT morphology variations between the groups, which we plan to report in a future paper.

Contributors SLR, SAQ and LLL contributed to the conception or design of work. MC, SSS, SW, SAQ and LLL contributed to the data collection. SLR, RK and LLL contributed to the data analysis and/or interpretation. All authors contributed to the drafting of the article and critical review of the article.

Funding This study has received funding from the Royal Victorian Eye and Ear Hospital Grants Program 2013-2014 (Melbourne), Diabetes Australia Research Program Grant 2015 (Canberra), Ramaciotti Health Investment Grant 2016 (Sydney) and the Hazel Jean Eastham Bequest (Melbourne). Centre for Eye Research Australia receives operational infrastructure support from the Victorian government. Associate Professor Lim (GNT 1109330) and Dr Wickremasinghe (GNT 1128343) are supported by National Health and Medical Research Council (NHMRC) Early Career Fellowships. The sponsor or funding organisations had no role in the design or conduct of the study; collection, management, analysis and interpretation of the data; preparation, review or approval of the manuscript or decision to submit the manuscript for publication. Dr Kandasamy and Associate Professor Lim had full access to all the data in the study and take responsibility for the integrity of the data and the accuracy of the data analysis.

Competing interests A/Prof Lim has received consultancy fees from Abbvie, Allergan and Bayer, and her institution has received research funding from Bayer that does not pertain to this study.

Patient consent for publication Obtained.
Ethics approval The trial received approval from the Human Research Ethics Committee of the RVEEH.

Provenance and peer review Not commissioned; externally peer reviewed.

Data sharing statement The trial data includes confidential patient medical information and patient informed consent was not obtained for the purpose of storing such data onto an online repository for public access.

\section{ORCID iDs}

Rathika Kandasamy http://orcid.org/0000-0002-5415-158X

Sophie L Rogers http://orcid.org/0000-0001-8114-7773

\section{REFERENCES}

1 Mitchell P, Annemans L, Gallagher M, et al. Cost-effectiveness of ranibizumab in treatment of diabetic macular oedema (DME) causing visual impairment: evidence from the restore trial. Br J Ophthalmol 2012:96:688-93.

2 Dowler Jet al. The natural history of macular edema after cataract surgery in diabetes. Ophthalmology 1999;106:663-8.

3 Krepler K, Biowski R, Schrey S, et al. Cataract surgery in patients with diabetic retinopathy: visual outcome, progression of diabetic retinopathy, and incidence of diabetic macular oedema. Graefe's Arch Clin Exp Ophthalmol 2002;240:735-8.

4 Zaczek A, Olivestedt G, Zetterström C. Visual outcome after phacoemulsification and IOL implantation in diabetic patients. Br J Ophthalmol 1999;83:1036-41.

5 Bressler SB, Baker CW, Almukhtar T, et al. Pilot study of individuals with diabetic macular edema undergoing cataract surgery. JAMA Ophthalmol 2014;132:224-6.

6 Patel Jl, Hykin PG, Cree IA. Diabetic cataract removal: postoperative progression of maculopathy--growth factor and clinical analysis. Br J Ophthalmol 2006;90:697-701.

7 Funatsu H, Noma H, Mimura T, et al. Association of vitreous inflammatory factors with diabetic macular edema. Ophthalmology 2009;116:73-9.

8 Imai S, Otsuka T, Naito A, et al. Triamcinolone acetonide suppresses inflammation and facilitates vascular barrier function in human retinal microvascular endothelial cells. Curr Neurovasc Res 2017;14.

9 Kriechbaum K, Prager S, Mylonas G, et al. Intravitreal bevacizumab (Avastin) versus triamcinolone (Volon a) for treatment of diabetic macular edema: one-year results. Eye 2014;28:9-16.

10 Aksoy S, Yilmaz G, Akkoyun I, et al. Comparison of intravitreal bevacizumab and triamcinolone acetonide theraphies for diffuse diabetic macular edema. Int J Ophthalmol 2015:8:550-5.

11 Shimura M, Nakazawa T, Yasuda K, et al. Comparative therapy evaluation of intravitreal bevacizumab and triamcinolone acetonide on persistent diffuse diabetic macular edema. Am J Ophthalmol 2008;145:854-61.

12 Isaac DLC, Abud MB, Frantz KA, et al. Comparing intravitreal triamcinolone acetonide and bevacizumab injections for the treatment of diabetic macular oedema: a randomized double-blind study. Acta Ophthalmol 2012;90:56-60.

13 Lim LL, Morrison JL, Constantinou M, et al. Diabetic Macular Edema at the time of Cataract Surgery trial: a prospective, randomized clinical trial of intravitreous bevacizumab versus triamcinolone in patients with diabetic macular oedema at the time of cataract surgery - preliminary 6 month results. Clin Exp Ophthalmol 2016:44:233-42.

14 JWR T. Applied longitudinal data analysis for epidemiology: a practical guide. 2nd edn Cambridge: Cambridge University Press, 2013.

15 Habib MS, Cannon PS, Steel DHW. The combination of intravitreal triamcinolone and phacoemulsification surgery in patients with diabeticfoveal oedema and cataract. BMC Ophthalmol 2005;5.

16 Lam DSC, Chan CKM, Mohamed S, et al. Phacoemulsification with intravitreal triamcinolone in patients with cataract and coexisting diabetic macular oedema: a 6-month prospective pilot study. Eye 2005;19:885-90.

17 Takata C, Messias A, Folgosa MS, et al. Intravitreal injection versus subtenon infusion of triamcinolone acetonide during cataract surgery in patients with refractory diabetic macular edema. Retina 2010:30:562-9.

18 Lanzagorta-Aresti A, Palacios-Pozo E, Menezo Rozalen JL, et al. Prevention of vision loss after cataract surgery in diabetic macular edema with intravitreal bevacizumab. Retina 2009;29:530-5.

19 Takamura Y, Kubo E, Akagi Y. Analysis of the effect of intravitreal bevacizumab injection on diabetic macular edema after cataract surgery. Ophthalmology 2009;116:1151-7.

20 Chen CH, Liu YC, Wu PC. The combination of intravitreal bevacizumab and phacoemulsification surgery in patients with cataract and coexisting diabetic macular edema. J Ocul Pharmacol Ther 2009;25:83-90.

21 Sohn HJ, Han DH, Kim IT, et al. Changes in aqueous concentrations of various cytokines after intravitreal triamcinolone versus bevacizumab for diabetic macular edema. Am J Ophthalmol 2011;152:686-94.

22 Roh MI, Kim HS, Song JH, et al. Effect of intravitreal bevacizumab injection on aqueous humor cytokine levels in clinically significant macular edema. Ophthalmology 2009;116:80-6.

23 Dong N, Xu B, Chu L, et al. Study of 27 aqueous humor cytokines in type 2 diabetic patients with or without macular edema. PLoS One 2015;10:e0125329. 
24 Jonas JB, Jonas RA, Neumaier M, et al. Cytokine concentration in aqueous humor of eyes with diabetic macular edema. Retina 2012;32:2150-7.

25 Maturi RK, Glassman AR, Liu D, et al. Effect of adding dexamethasone to continued ranibizumab treatment in patients with persistent diabetic macular edema: a DRCR network phase 2 randomized clinical trial. JAMA Ophthalmol 2018;136:29-38.

26 Joe AW, Wickremasinghe SS, Gillies MC, et al. Dexamethasone implant for the treatment of persistent diabetic macular oedema despite long-term treatment with bevacizumab. Clin Exp Ophthalmol 2018. [Epub ahead of print: 06 Aug 2018].

27 Kim BY, Smith SD, Kaiser PK. Optical coherence tomographic patterns of diabetic macular edema. Am J Ophthalmol 2006;142:405-12.

28 Browning DJ, Glassman AR, Aiello LP, et al. Relationship between optical coherence tomography-measured central retinal thickness and visual acuity in diabetic macular edema. Ophthalmology 2007;114:525-36.

29 Murakami T, Yoshimura N. Structural changes in individual retinal layers in diabetic macular edema. J Diabetes Res 2013;2013:1-11.
30 Alasil T, Keane PA, Updike JF, et al. Relationship between optical coherence tomography retinal parameters and visual acuity in diabetic macular edema. Ophthalmology 2010;117:2379-86.

31 Wong RLM, Lee JWY, Yau GSK, et al. Relationship between outer retinal layers thickness and visual acuity in diabetic macular edema. Biomed Res Int 2015:2015:1-5

32 Eliwa TF, Hussein MA, Zaki MA, et al. Outer retinal layer thickness as good visual predictor in patients with diabetic macular edema. Retina 2017.

33 Ahmadabadi HF, Mohammadi M, Beheshtnejad H, et al. Effect of intravitreal triamcinolone acetonide injection on central macular thickness in diabetic patients having phacoemulsification. J Cataract Refract Surg 2010;36:917-22.

34 VanderBeek BL, Bonaffini SG, Ma L. The association between intravitreal steroids and Post-Injection endophthalmitis rates. Ophthalmology 2015;122:2311-5.

35 Eadie BD, Etminan M, Carleton BC, et al. Association of repeated Intravitreous bevacizumab injections with risk for glaucoma surgery. JAMA ophthalmol 2017;135:363-8. 